\title{
Cytogenetic and Genome Research
}

Aitman, T.J. 161

Al-Sukhni, W. 211

Antonarakis, S.E. 7

Armengol, L. 108

Aston, E. 94

Aten, E. 313

Auer, H. 278

Azam, T. 65

Bailey, D.K. 94

Bailey, J.A. 234

Bauters, M. 44

Bayrak-Toydemir, P. 94

Beckmann, J.S. 7

Birmingham, D.J. 131

Blakemore, A.I. 17

Breuning, M.H.B. 313

Brothman, A.R. 94

Bruhn, L. 94

Burwinkel, B. 183

Buysse, K. 176

Chen, J.M. 102

Chong, K. 65

Chung, E.K. 131

Cooper, D.N. 5, 288

Craig, J.M. 270

Cutler, G. 297

De Preter, K. 176

de Smith, A.J. 17

den Dunnen, J.T. 313

den Hollander, N.S. 65

Edberg, J.C. 142

Eichler, E.E. 234

Estivill, X. 108

Fanciulli, M. 161

Fawcett, C. 79

Férec, C. 102

FitzPatrick, D. 65

Froguel, P. 17

Froyen, G. 44

Füst, G. 118

Gallinger, S. 211

Geurts van Kessel, A. 188

Goobie, S. 65

Gu, W. 54

Hannes, F. 88

Harvard, C. 79

Hebbring, S.J. 205

Hegele, R.A. 169

Herbert, L.A. 131

Holden, J.J.A. 79
Hollox, E.J. 148

Holmes, C.C. 307

Hoogerbrugge, N. 188

Hopkins, C. 94

Ingelman-Sundberg, M. 195

Jeon, J.T. 333

Jobling, M.A. 253

Johansson, I. 195

Kalf, M.E. 313

Kassner, P.D. 297

Katayama, H. 224

Kaur, G. 156

Kehrer-Sawatzki, H. 5, 288

Kelley, J.M. 142

Kidd, J.M. 234

Kimura, A. 156

Kitzmiller, K.J. 131

Knijnenburg, J. 65

Kodaira, M. 224

Kriek, M. 313

Kuiper, R.P. 188

Kumps, C. 176

Kusenda, M. 36

Lachman, H.M. 27

Lanktree, M. 169

Le Maréchal, C. 102

Lee, J.H. 333

Lewis, M.E.S. 79

Li, X. 142

Ligtenberg, M.J.L. 188

Lionel, A.C. 65

Liu, X. 79

Lupski, J.R. 54

Maher, E. 65

Marshall, C.R. 65

Marynen, P. 44

Masson, E. 102

Mehra, N. 156

Mendoza-Londono, R. 65

Menten, B. 176

Mitchell, S. 94

Monaco, A.P. 322

Moyer, A.M. 205

Nagaraja, H.N. 131

Nakajima, T. 156

Nei, M. 263

Nevelsteen, J. 44

Newbury, D.F. 322

Notini, A.J. 270

Nozawa, M. 263
Perry, G.H. 283

Peters, G. 94

Poppe, B. 176

Ptacek, T. 142

Qiao, Y. 79

Rabionet, R. 108

Ragoussis, J. 322

Rajcan-Separovic, E. 79

Riendeau, N. 79

Riethman, H. 244

Rovin, B.H. 131

Ruivenkamp, C. 65, 313

Satoh, Y. 224

Savelli, S.L. 131

Scherer, S.W. 65

Sebat, J. 36

Shago, M. 65

Shah, S.P. 343

Sharkey, F.H. 65

Sharp, A.J. 7

Speleman, F. 176

Szilágyi, Á. 118

Szuhai, K. 65, 313

Takahashi, N. 224

Tanke, H.J. 65

Tchatchou, S. 183

Thygesen, H.H. 313

Tsalenko, A. 94

Tsang, P. 94

Tsao, B.P. 131

Van Esch, H. 44

Vandewalle, J. 44

Venkatachalam, R. 188

Vermeesch, J.R. 88

Vossen, R.H.A.M. 313

Vyse, T.J. 161

Walters, R.G. 17

Weinshilboum, R.M. 205

Weuts, A. 44

Whitby, H. 94

White, S.J. 270, 313

Winchester, L. 322

Wintle, R.F. 65

Wu, Y.L. 131

Yang, Y. 131

Yau, C. 307

Yu, C.Y. 131

Zhou, B. 131 